\title{
Importations, diffusions et inflexions des Legal Consciousness Studies dans la recherche française
}

Vincent-Arnaud Chappe, CSI i3 - Centre de Sociologie de l'Innovation i3

Jérôme Pélisse, CSO, Centre de sociologie des organisations

Anna Egea, CSO, Centre de sociologie des organisations

Version auteur, manuscrit accepté (envoyé le 13 juin 2018)

Article publié dans Droit et Société, 2018, 2018/3 (N 100), p. 665-684.

Référence de la publication :

Chappe Vincent-Arnaud, Pélisse Jérôme, Egea Anna, «Importations, diffusions et inflexions des Legal Consciousness Studies dans la recherche française », Droit et société, 2018/3 ( $\left.N^{\circ} 100\right)$, p. 665-684. DOI : 10.3917/drs1.100.0665. URL :

https://www.cairn.info/revue-droit-et-societe-2018-3-page-665.htm

\section{Résumé :}

Cet article propose un bilan critique de l'importation et des usages des legal consciousness studies dans l'espace académique francophone. Ce cadre d'analyse a révolutionné pour une part la sociologie du droit américaine dans les années 1990 et 2000, en mettant l'accent sur les acteurs profanes, leurs représentations ordinaires et usages quotidiens du droit. Vingt ans après la publication de l'ouvrage emblématique de cette perspective - The Common Place of Law. Stories of Everyday Life par P. Ewick et S. Silbey en 1998 -, quel état des lieux concernant le processus d'importation, de diffusion et d'inflexions des legal consciousness studies en France? Ont-elles contribué à renouveler les recherches en sociologie du droit, et plus généralement en sciences sociales? L'article s'appuie sur une base de données de citations d'une part, et l'analyse qualitative d'une vingtaine d'articles se référant aux legal consciousness studies, avant de proposer quelques pistes de recherche futures.

Mots-clés : legal consciousness, importation, traduction, hégémonie du droit 
Cet article propose un bilan critique de l'importation et des usages des legal consciousness studies (LCS) dans l'espace académique francophone. Ce cadre d'analyse a révolutionné pour une part la sociologie du droit américaine dans les années 1990 et 2000 en mettant l'accent sur les acteurs profanes, leurs représentations ordinaires et usages quotidiens du droit. Vingt ans après la publication de l'ouvrage emblématique The Common Place of Law. Stories of Everyday Life par P. Ewick et S. Silbey ${ }^{1}$, les legal consciousness studies ont-elles contribué à renouveler les recherches en sociologie du droit, et plus généralement en sciences sociales en France? Ont-elles participé au renforcement d'une sociologie du droit centrée sur l'expérience quotidienne de la légalité, dans sa dimension structurante de la réalité sociale? Sont-elles restées confinées dans la sociologie du droit ou ont-elles essaimé plus largement?

En se fondant sur l'analyse bibliométrique des citations d'un corpus de textes centraux de ce courant de recherche et de productions visant explicitement à les importer dans l'espace de recherche français, puis sur une analyse thématique de vingt articles se référant à l'un ou plusieurs de ces travaux, nous étudions le processus de diffusion des legal consciousness studies dans l'espace académique francophone.

Trois temps scandent cette diffusion. Un premier moment de traduction et d'importation s'est déroulé entre la fin des années 1990 et 2005, exclusivement dans le champ de l'anthropologie et de la sociologie du droit. Un deuxième temps a été celui d'une diffusion progressive, marqué par un usage déférent et superficiel, plus individualiste que structuraliste, avec une oblitération de la notion de legality qui lui est associée et des enjeux d'hégémonie du droit, pourtant essentiels dans ce courant de recherche. On voit aussi la multiplication des références au concept de legal consciousness dans des contextes principalement institutionnels d'usages du droit, voire du tribunal, loin des évolutions imprimées par Ewick et Silbey à cette perspective d'analyse. Un troisième moment, en cours, fait place à des appropriations à la fois plus fidèles (i.e., hors de tout contexte institutionnel, même si c'est encore le cas d'une minorité de recherches) et plus distanciées, qui mettent plus frontalement en débat la question des pouvoirs, éventuellement limités ou limitant, qu'offre ou que cadre le droit et la manière dont des acteurs, socialement situés, le construisent, le vivent et le font vivre dans leur quotidien. Après un rappel des origines de ce courant de recherche, l'article revient sur le processus d'importation (I) puis propose des éléments d'objectivation de sa diffusion en France (II). Il évoque ensuite la variété des travaux s'inscrivant dans ce champ de recherche, les renouvellements que la notion de legal consciousness a ouvert mais aussi les limites qu'a rencontrées sa diffusion et l'effervescence récente qui semble en cours (III). On propose en conclusion quelques pistes d'approfondissement de cet usage renouvelé des Legal Consciousness Studies dans les pays francophones.

\section{1) Revenir sur une importation : des legal consciousness à la conscience du droit}

La notion de conscience juridique ou conscience du droit pourrait apparaître comme un objet classique de la sociologie du droit. Elle constitue une entrée dans le Dictionnaire de sociologie juridique publié en $1993^{2}$, qui évoque des recherches comme celles d'A. Podgorocki qui dirige un programme international de grande ampleur entre 1968 et 1973 sur Knowledge and Opinion about Law, ou de F. Terré et A. Sayag publiant dans l'Année sociologique en 1975 un article intitulé "Connaissances et consciences du droit,

\footnotetext{
${ }^{1}$ Ewick Patricia et Silbey Susan S., 1998, The common place of law: Stories from everyday life, Chicago, University of Chicago Press.

${ }^{2}$ Arnaud A.-J., 1993, Dictionnaire encyclopédique de théorie et de sociologie du droit, Paris, LGDJ.
} 
problèmes de recherche $»^{3}$. L'analyse marxiste du droit, qui a aussi interrogé l'expérience, les connaissances et les consciences du droit à partir d'une critique de l'idéologie juridique est également rappelée. Ces propositions restent toutefois largement théoriques, qu'elles s'inscrivent dans des perspectives fonctionnalistes qui visent à appréhender le degré de connaissance du droit des citoyens ou dans des approches critiques qui ne s'appuient pas sur des enquêtes empiriques.

Dans cette dernière perspective, D. Trubek appelle, en 1984 puis en 1989 avec J. Esser 4 , à développer des travaux empiriques autour de la question des expériences juridiques quotidiennes d'acteurs ordinaires, moins dans une perspective de «besoins juridiques » que la puissance publique ou les professionnels du droit auraient à combler, que dans une ligne critique visant à déconstruire et à comprendre l'idéologie juridique qui règle la vie quotidienne et légitime des pouvoirs comme « allant de soi »5. Interprétables comme une forme de sociologisation des Critical legal studies ${ }^{6}$, des travaux empiriques sur les consciences du droit voient le jour et sont présentés dans les années 1980 dans le cadre d'un séminaire organisé à Amherst par A. Sarat et S. Silbey qui privilégiaient, comme le rappelle S. Silbey dans ce dossier, une entrée par la culture et l'idéologie. Il s'agissait d'étudier les pratiques concrètes de la vie quotidienne dans lesquelles les règles légales sont utilisées et perçues (ou non) comme des éléments constitutifs de la réalité, plutôt que les professionnels du droit ou comment fonctionnaient les tribunaux.

Le concept de Legal consciousness se développe au même moment en Europe et notamment en France, dans des travaux d'anthropologie davantage centrés sur les questions de socialisation juridique et sans cette perspective critique. C. KourilskyAugeven initie dès 1986 des enquêtes impliquant des comparaisons internationales avec les pays de l'Est centrées sur la caractérisation et l'analyse des modes de socialisation au droit pour approcher les rapports ordinaires au droit et les cultures juridiques populaires ${ }^{7}$. Les analyses s'intéressent aux perceptions et aux attitudes plus qu'aux conceptions et aux pratiques. Un premier point de rencontre s'opère quand P. Ewick et S. Silbey finalisent la publication de The Commonplace of law. En 1997, elles publient un article dans Droit et culture, la revue fondée par C. Kourilsky-Augeven, qui est la traduction du chapitre 3 de leur ouvrage qui sera publié l'année suivante ${ }^{8}$. En 1998, un numéro spécial de cette même revue porte sur «Legal consciousness et socialisation juridique : pour un dialogue franco-américain ». Il comporte un article de D. Engel et F. Munger, qui écriront plus tard un autre ouvrage important, Rights of Inclusion, publié en

\footnotetext{
3 Sayag A. et Terré F., 1975, "Connaissance et conscience du droit: Problèmes de recherche ", L'Année sociologique, vol. 26, p. 465-495.

4 Trubek D. M., 1984, "Where the action is: critical legal studies and empiricism », Stanford Law Review, 36, 575-622 ; Trubek D.M. et Esser J., 1989, «"Critical Empiricism” in American Legal Studies: Paradox, Program, or Pandora's Box? », Law \& Social Inquiry, 14 (1), p. 3-52.

5 Voir aussi Engel D.M., 1998, « How Does Law Matter in the Constitution of Legal Consciousness? », in B. G. Garth and A. Sarat (Eds), How Does Law Matter? Evanston, Northwestern University Press, 1998, p. 109145. Analysant six recherches sur ces questions, l'auteur retrace cette double origine des legal consciousness studies américaines, entre approches fonctionnalistes et plus critiques.

6 Pélisse J., 2009, «Les Legal Consciousness Studies: une sociologisation domestiquée des Critical Legal Studies? » in Bentouhami H., Kupiec A., Grangé N. (dir.), Le souci du droit : où en est la théorie critique? Tonka, p. 223-238.

7 Kourilsky C., 1986, « Connaissances et représentations du droit », Droit et société, vol. 4 (1), p. 383-403 ; Id., 2004, Images and uses of law among ordinary people, Société de Législation Comparée.

${ }^{8}$ Une première rencontre s'était déjà opérée: S. Silbey publie en effet un article dans un dossier de Droit et société en 1991, coordonné par C. Kourilsky, qui portait sur « Le rapport des jeunes au Droit à l'Est et à l'Ouest ». Le titre de l'article de Silbey incluait déjà la notion de conscience juridique, puisqu'il s'intitulait «Un jeu d'enfant : une analyse culturelle de la conscience juridique des adolescents américains ».
} 
2003. Une première tentative d'importation a donc lieu dès la fin des années 1990, s'adressant à un public d'anthropologues du droit en France. C'est un échec, comme l'analyse bibliométrique l'objectivera: presque vingt ans après, les deux articles américains ne sont jamais cités d'après Google scholar ${ }^{9}$ !

Cette tentative est redoublée par une seconde quelques années plus tard. Découvrant l'ouvrage de Ewick et Silbey en 2001 lors d'un colloque organisé par Law and Society Association, L. Israël propose aux étudiants de l'ENS Cachan de traduire le même chapitre de l'ouvrage d'Ewick et Silbey que la traduction effectuée par C. Kourilsky, ce dont s'aperçoivent tardivement L. Israël et J. Pélisse, qui rédigent l'introduction présentant leur traduction (Terrains et travaux, 2004) ${ }^{10}$. J. Pélisse utilise quant à lui ces travaux dans son travail de thèse et publie dans Droit et Société en 2003 un article les utilisant pour analyser la mise en œuvre des $35 \mathrm{~h}$. Dans ce même numéro de Droit et société, un article en anglais de M. Garcia-Villegas présente d'ailleurs également les LCS de manière critique ${ }^{11}$. Cette première phase de découverte des legal consciousness studies se clôt en 2005 par une nouvelle publication de J. Pélisse dans Genèses ${ }^{12}$. Une seconde phase s'ouvre alors.

\section{2) Objectiver la diffusion d'une perspective de recherche}

Par une analyse bibliométrique, complétée par une analyse qualitative de la littérature recueillie, on peut alors objectiver les parcours d'importation de travaux et concepts associés aux LCS dans l'espace francophone.

\subsection{Une recherche bibliométrique : méthode, données, choix}

La diffusion d'un corpus de documents-cibles est analysée via la répartition des citations francophones qui en sont faites. On développe moins une analyse bibliométrique qui permettrait d'évaluer les chercheurs ${ }^{13}$ qu'une étude des modes de sociabilité scientifique approchés par les pratiques de citation ${ }^{14}$. Une note récente de L. Pinto évoque l'intérêt d' " une sociologie des citations », et souligne la nécessité d'objectiver la compétence citationnelle qui la sous-tend ${ }^{15}$. Par ailleurs précurseur méconnu des LCS en France ${ }^{16}$, L. Pinto émet "l'hypothèse que, de façon générale, l'acte de citer reflète

\footnotetext{
${ }^{9}$ L'article de Engel et Munger est en réalité cité une fois dans un article de I. Carles paru en 2010 dans Champs psy intitulé « Usage du droit et discriminations multiples : le genre des plaintes visant à lutter contre les discriminations raciales ».

10 Voir Israël L. et Pélisse J., 2004, «Quelques éléments sur les conditions d'une «importation » (Note liminaire à la traduction du texte de S. Silbey et P. Ewick)», Terrains \& travaux, n6, p. 101-111. La découverte de l'existence de la traduction de 1997 et du numéro spécial de Droit et culture en 1998 s'effectue après le séminaire de traduction organisé à Cachan. Une comparaison des choix de traduction, évoquée dans cette introduction, montre qu'on passe de la conscience juridique (chez Kourilsky) à la conscience du droit (chez les traducteurs cachanais), tandis que «before the law» est traduit non plus « devant la loi » mais « face au droit».

11 Voir Pélisse J., 2003, «Consciences du temps et consciences du droit chez des salariés à 35 heures » et Garcia-Villegas M., «Symbolic Power Without Symbolic Violence? Critical Comments on Legal Consciousness Studies in USA», Droit et Société, n53, p. 163-186 et 137-163. L'article de M. GarciaVillegas est la reprise d'un article publié dans la Florida Law Review la même année.

12 Pélisse J., « A-t-on conscience du droit? », op.cit.

13 Pontille D. et Torny D., 2013, « La manufacture de l'évaluation scientifique », Réseaux, vol. 1, p. 23-61.

14 Milard B., «Quelles sociabilités derrière les références bibliographiques? Citations et relations sociales », Socio-logos [En ligne], 8, 9 janvier 2014.

${ }^{15}$ Pinto L., « Note pour une sociologie des citations », Lire les sciences sociales, 16 avril 2015. https://llss.hypotheses.org/793 [Consulté le : 12 mars 2018].

${ }_{16}$ Pinto L., 1989, « Du "pépin" au litige de consommation. Une étude du sens juridique ordinaire », Actes de la recherche en sciences sociales. Vol. 76-77, p. 65-81.
} 
(objectivement aussi bien que subjectivement) la position occupée dans le champ, position qui commande la sélection et la hiérarchisation des textes et des auteurs ${ }^{17}$. Dans notre cas, c'est un usage plus rudimentaire que nous proposons des pratiques de citation visant à observer la diffusion d'un corpus de textes centraux des legal consciousness studies. Appuyé sur notre connaissance approfondie du champ Law and Society depuis quinze ans, ce corpus se constitue de sept productions scientifiques américaines et de six articles ou traductions en français qui ont souhaité jouer un rôle d'intermédiaire dans l'importation des LCS.

Les sept références américaines considérées comme centrales dans le champ des LCS

\begin{tabular}{|c|c|c|c|}
\hline Titre de la référence & Date & $\begin{array}{l}\text { Raisons de l'inclusion dans le } \\
\text { corpus }\end{array}$ & $\begin{array}{l}\text { Nombre de } \\
\text { citations en } \\
\text { français } \\
\text { (avril 2017) }\end{array}$ \\
\hline $\begin{array}{l}\text { Sarat A., " The law is "all over": Power, } \\
\text { Resistance and the Legal Consciousness of } \\
\text { the Welfare Poor », Yale Law Journal of } \\
\text { Humanities, vol. } 2\end{array}$ & 1990 & $\begin{array}{l}\text { Un article fondateur des LCS, où } \\
\text { l'auteur hésite entre la notion de } \\
\text { legal ideology et legal } \\
\text { consciousness. }\end{array}$ & 14 \\
\hline $\begin{array}{l}\text { Merry S.E., Getting Justice and Getting Even, } \\
\text { Legal Consciousness Among Working-Class } \\
\text { Americans, Univ of Chicago Press }\end{array}$ & 1990 & $\begin{array}{l}\text { Ouvrage fondateur des LCS } \\
\text { appuyé sur une enquête au sein } \\
\text { de tribunaux de première } \\
\text { instance }\end{array}$ & 30 \\
\hline $\begin{array}{l}\text { Mc Cann M. W., Rights at Work. Pay equity } \\
\text { reform and the politics of legal mobilization, } \\
\text { Univ of Chicago Press }\end{array}$ & 1994 & $\begin{array}{l}\text { Ouvrage central du champ Law } \\
\text { and society qui articule sociologie } \\
\text { des mouvements sociaux et des } \\
\text { LCS }\end{array}$ & 48 \\
\hline $\begin{array}{l}\text { Ewick P., Silbey S.S., The common place of } \\
\text { law: Stories from everyday life, Univ. of } \\
\text { Chicago Press }\end{array}$ & 1998 & Ouvrage séminal des LCS & 65 \\
\hline $\begin{array}{l}\text { Engle D., Munger F., Rights of Inclusion. Law } \\
\text { and identity in the life stories of Americans } \\
\text { with disabilities, Univ of Chicago Press }\end{array}$ & 2003 & $\begin{array}{l}\text { Autre ouvrage central des LCS, } \\
\text { traduit en français en } 2017\end{array}$ & 9 \\
\hline $\begin{array}{l}\text { Silbey S.S., "After legal consciousness », } \\
\text { Annual Review of Law and Social Science, 1, } \\
\text { p. 323-368 }\end{array}$ & 2005 & $\begin{array}{l}\text { Retour de S. Silbey sur les usages } \\
\text { et mésusages du concept de legal } \\
\text { consciousness dans la sociologie } \\
\text { du droit américaine }\end{array}$ & 6 \\
\hline $\begin{array}{l}\text { Nielsen L.B., Fleury-Steiner B. (eds), The } \\
\text { New Civil Rights Research: A Constitutive } \\
\text { Approach, Ashgate, 2006. Préface de M. } \\
\text { McCann }\end{array}$ & 2006 & $\begin{array}{l}\text { Ouvrage issu de communications } \\
\text { présentées dans le réseau LCS des } \\
\text { colloques Law and society des } \\
\text { années précédentes, évoquant } \\
\text { divers mondes sociaux }\end{array}$ & 0 \\
\hline
\end{tabular}

Les six productions francophones traduisant ou présentant les LCS

\begin{tabular}{|l|l|l|l|}
\hline Titre de la référence Date & $\begin{array}{l}\text { Raisons de l'inclusion dans le } \\
\text { corpus }\end{array}$ & $\begin{array}{l}\text { Nombre de } \\
\text { citations en } \\
\text { français (avril } \\
\text { 2017) }\end{array}$ \\
\hline $\begin{array}{l}\text { Silbey S., Ewick P., «Devant la loi: la } \\
\text { construction sociale du juridique » in C. } \\
\text { Kourilsky-Augeven (dir.) Socialisation }\end{array}$ & $\begin{array}{l}\text { Première traduction du chapitre } \\
\text { Silbey de l'ouvrage de Ewick et } \\
\text { Sils }\end{array}$ & 8 \\
\hline
\end{tabular}

17 Pinto L., « Note pour... », op.cit., p. 2.

18 L'article de Silbey de 1991 n'a pas été retenu parce qu'il ne présente pas le courant des legal consciousness studies, encore en cours d'élaboration aux Etats-Unis. L'article de Garcia-Villegas publié en 


\begin{tabular}{|c|c|c|c|}
\hline \multicolumn{4}{|l|}{$\begin{array}{l}\text { juridique et conscience du droit: attitudes } \\
\text { individuelles, modèles culturels et } \\
\text { changement social. Paris, MSH-LGDJ, p. 33- } \\
56 .\end{array}$} \\
\hline $\begin{array}{l}\text { Kourilsky C., "A case study of legal } \\
\text { consciousness : Legal Consciousness et } \\
\text { sociologie juridique : pour un dialogue } \\
\text { franco-américain », Droit et culture }{ }^{\circ} 35 \text {, } \\
\text { pp. } 23-41 \text {. }\end{array}$ & 1998 & $\begin{array}{l}\text { Article présentant les LCS aux } \\
\text { chercheurs francophones }\end{array}$ & 0 \\
\hline $\begin{array}{l}\text { Pélisse J., "Consciences du temps et } \\
\text { consciences du droit chez des salariés à } \\
35 \mathrm{~h} \text { », Droit et société } \mathrm{n}{ }^{\circ} 53 \text {. }\end{array}$ & 2003 & $\begin{array}{l}\text { Article qui présente, utilise et } \\
\text { propose une série de critiques } \\
\text { des LCS à partir d'un terrain } \\
\text { centré sur la mise en œuvre des } \\
\text { 35h en France }\end{array}$ & 11 \\
\hline $\begin{array}{l}\text { Israël L., Pélisse J., «Quelques éléments sur } \\
\text { les conditions d'une «importation» (Note } \\
\text { liminaire à la traduction du texte de S. } \\
\text { Silbey et P. Ewick) », Terrains \& travaux, 6, } \\
\text { p. 101-111. }\end{array}$ & 2004 & 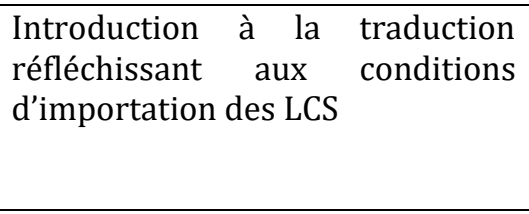 & 4 \\
\hline $\begin{array}{l}\text { Ewick P., Silbey S.S., " La construction } \\
\text { sociale de la légalité », Terrains et travaux, } \\
\text { 6, p. 112-138 (traduction : G. Cassan, D. } \\
\text { Didier, E. Gardella, L. Israël, R. Lutaud, C. } \\
\text { Ollivier, J. Pélisse, M. Pujuguet, J. } \\
\text { Souloumiac, M. Trespeuch, G. Truc, B. } \\
\text { Williams). }\end{array}$ & 2004 & $\begin{array}{l}\text { Seconde traduction du chapitre } 3 \\
\text { de l'ouvrage de Ewick et Silbey, } \\
\text { par les étudiants de l'ENS Cachan }\end{array}$ & 12 \\
\hline $\begin{array}{l}\text { Pélisse J., "A-t-on conscience du droit? } \\
\text { Autour des legal consciousness studies", } \\
\text { Genèses } \mathrm{n}^{\circ} 59 / 2 .\end{array}$ & 2005 & $\begin{array}{l}\text { Article présentant, évoquant } \\
\text { certaines limites et réfléchissant } \\
\text { aux conditions d'importation des } \\
\text { LCS }\end{array}$ & 54 \\
\hline
\end{tabular}

Pour chacun de ces documents, nous avons identifié l'ensemble des citations francophones ${ }^{19}$. Aucune base de données ne permettait de réaliser ce travail de manière totalement satisfaisante: les grandes bases américaines n'incluent que très peu de revues francophones, et les bases de données françaises (Cairn, Isidore) ne permettent pas de remonter des documents cibles à ceux qui les citent. Nous utilisons donc Google scholar tout en étant conscients de ses limites ${ }^{20}$. Grâce à l'outil Publish or Perish, l'ensemble des références bibliographiques francophones citant au moins l'un de ces 13 documents a été identifié, permettant une visualisation des réseaux et pôles de citations.

\subsection{Résultats : rythmes, supports, nœuds}

Une diffusion plutôt lente et régulière des LCS

Les différents documents sont cités de façon très inégale. The Common Place of Law est cité 65 fois, suivi par Rights at Work (48 citations) puis Getting Justice (30 citations)21. Le nombre de ces citations augmente nettement à partir de la fin des années 2000.

2003 n'a pas été inclus car il est publié en anglais, même s'il est repris dans une revue française. De fait, il est cité 8 fois, dont 5 fois en français dans Google scholar.

${ }^{19}$ Les travaux québécois sont donc inclus mais ces derniers ne citent jamais les LCS, un phénomène déjà observé à propos d'autres courants américains de sociologie du droit comme le cause lawyering.

20 Sur ces limites mais aussi celles d'autres bases de données, voir Durand-Barthez M. et al., 2009, « Outils et méthodes », Documentaliste-Sciences de l'Information, vol. 46 (4), p. 44-59.

${ }^{21}$ Ces chiffres sont proches, en niveau, avec ceux qui citent deux ouvrages majeurs du champ STS qui s'est développé une dizaine d'années auparavant (en l'occurrence Social Shaping of Technology, de McKenzie et Wacjman, réédité en 1999, après une première édition en 1985) et The Scientific Revolution de S. Shapin publié en 1996. 


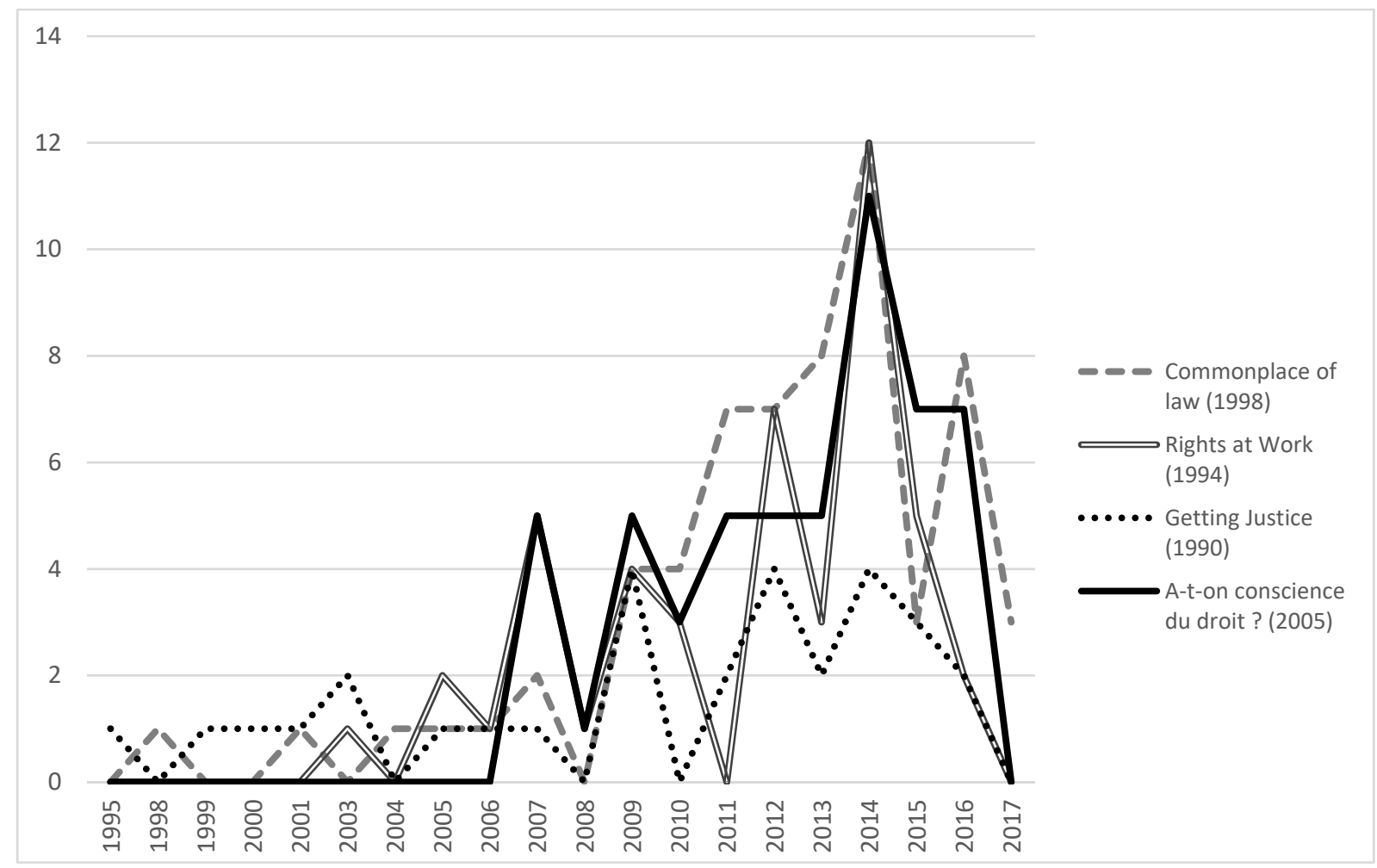

Lecture : en 2014, The Commonplace of Law et Rights at Work ont été cités douze fois parmi les publications francophones présentes dans Google Scholar. L'année 2017 n'est pas significative puisque la base de données a été constituée en avril 2017.

La diffusion des LCS en France se réalise dix ans après la publication de l'ouvrage emblématique d'Ewick et Silbey, et trois à quatre ans après la traduction dans Terrains et travaux et la présentation du courant de recherche par J. Pélisse en 2005. Cette présentation est bien plus citée que la traduction du chapitre 3 de The Common Place of Law (cité douze fois en douze ans, entre 2005 et 2016). La traduction réalisée en 1997 par C. Kourilsky-Augeven de ce même chapitre n'a rencontré que peu d'écho en termes de citations (huit entre 1997 et 2017), et les articles en anglais, même publiés dans des revues françaises, sont très rarement cités. Pour se diffuser et être cités, les textes doivent aussi être publiés dans un support de diffusion adapté. Ainsi, l'introduction proposée par C. Kourilsky dans le dossier qu'elle coordonne en 1998 n'est jamais cité : le support est local (la revue Droit et culture est éditée à l'Université Paris X Nanterre) et ancré dans une sous-discipline (l'anthropologie juridique) regroupant un petit nombre de chercheurs. En revanche la synthèse proposée par J. Pélisse dans Genèses en 2005 est régulièrement citée: publiée dans une revue au plus large auditoire qui s'inscrit au croisement de différents champs disciplinaires (sociologie, histoire, anthropologie).

\section{Les revues et l'ancrage disciplinaire}

33 des 175 articles faisant référence à au moins un document cible ont été publiés dans Droit et Société, soit $19 \%$. Cette revue a donc largement joué un rôle d'accueil pour des travaux s'inspirant des LCS. Onze articles ont été publiés dans Genèses et dix dans L'année sociologique (principalement à l'occasion d'un numéro spécial). D’autres revues généralistes ont également participé à la diffusion, puisque neuf articles ont été publiés dans Sociologie du travail, et sept dans la Revue française de science politique. La Revue française de sociologie y fait écho plus tardivement, et dans une moindre mesure. La 
diffusion dans les revues francophones a d'abord été sectorielle, avant une forme de banalisation assez récente dans les autres domaines de la sociologie française.

Un pôle de citation autour de l'ENS Cachan

Du côté des auteurs citants, une polarisation autour d'un noyau d'auteurs qui citent un document cible à plusieurs reprises dans différentes publications est nettement visible. A côté de ces auteurs inscrits dans la sociologie du droit, de nombreux autres chercheurs ont une pratique de citation unique. Si on regarde les documents les plus cités :

\begin{tabular}{|l|c|c|}
\hline Document-cible & $\begin{array}{l}\text { Nombre d'auteurs qui ne le } \\
\text { citent qu'une fois }\end{array}$ & $\begin{array}{l}\text { Nombre d'auteurs qui le citent } \\
\text { plus d'une fois }\end{array}$ \\
\hline The Common Place of Law & 61 & 13 \\
\hline «A-t-on conscience du droit?» & 47 & 15 \\
\hline Rights at Work & 35 & 14 \\
\hline
\end{tabular}

La visualisation en réseau permet de repérer facilement ce noyau de citants qui ont une pratique de référence à de multiples publications en lien avec les LCS (voir graphique). 


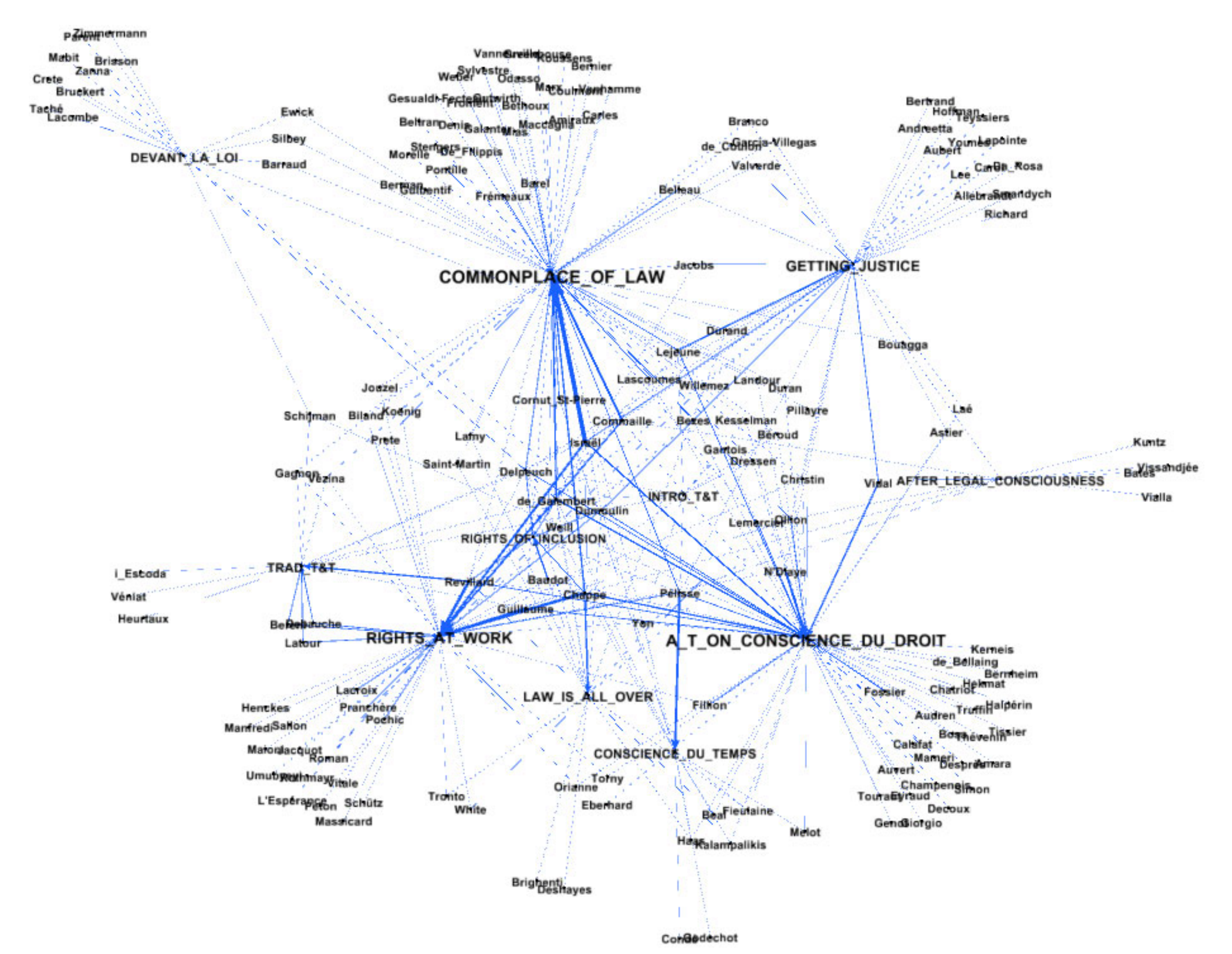


Le graphique permet de visualiser les rapports de citation : les documents-cibles sont écrits en majuscule, les traits représentent les pratiques de citation des auteur.e.s vers les documents identifiés, la grosseur du trait étant proportionnelle au nombre de citations d'un.e auteur.e vers la cible. Les auteurs multi et pluri-citants se trouvent au centre du graphique. Soit ils ont participé directement à l'importation des LCS en France (L. Israël, J. Pélisse), soit ils ont un lien très clair avec l'ENS Cachan, voire plus particulièrement avec l'ISP-Institut des sciences sociales du politique (J. Commaille, C. de Galembert, T. Delpeuch, L. Dumoulin, M. Ndiaye, V.-A. Chappe, A. Revillard, etc.). Ce laboratoire de sociologie du droit et de l'action publique a contribué à la mise en visibilité sinon l'appropriation (et aujourd'hui la mise en discussion) des travaux LCS en France, notamment par l'invitation de S. Silbey à plusieurs séminaires, celle-ci ayant d'ailleurs reçu le doctorat honoris causa de l'ENS Cachan en 2007. En lien avec ce laboratoire, un autre sous-pôle se détache, plus proche de la science politique (P. Lascoumes, P. Duran, A. Lejeune, ainsi que P.Y. Baudot ou E. Weill), ainsi qu'un troisième, composé de chercheurs non liés à l'ISP et l'ENS Cachan et étudiant avant tout le droit du travail et les relations professionnelles (L. Willemez, S. Béroud, M. Gantois, C. Guillaume, M. Dressen, D. Kesselman). Cette situation peut expliquer le triple ancrage des LCS en France, entre sociologie politique du droit, sociologie de l'action publique et sociologie du (droit du) travail.

\section{Dépasser la bibliométrie}

Ces éléments objectivent un mode de diffusion, des pôles et des canaux (des traductions mais aussi des articles de présentation), une temporalité (un effet retard mais aussi un pic en 2014) et des supports (une revue spécialisée et, progressivement, des revues plus généralistes). Ces premiers éléments restent malgré tout très limités, cette approche bibliométrique laissant de côté communications et thèses et ne disant rien des usages effectifs des LCS dans les travaux francophones.

\section{3) D’un usage limité et superficiel à une effervescence récente autour des legal consciousness}

Après une lecture attentive de vingt des travaux citant les legal consciousness studies, notre analyse montre un usage limité, sinon distant, de ces références, du moins dans un premier temps, ce qui rejoint le constat de S. Silbey en 2005 pour l'espace américain ${ }^{22}$. Toutefois, une effervescence récente semble montrer un intérêt renouvelé pour cette perspective de recherche en France, visant davantage à la compléter qu'à l'utiliser avec déférence, sinon à la discuter plus frontalement comme ce dossier de Droit et Société en témoigne.

\subsection{Une approche déférente et distante aux Legal consciousness studies}

\section{Une analyse de contenu systématique}

Un sous-corpus de vingt articles repérés dans notre base de données initiale (citant au moins un des articles ou ouvrages de référence) a été élaboré en faisant varier les auteurs, le document cible auquel ils se réfèrent, le type de terrains, les périodes concernées et les revues. Ces articles sont référencés en annexe, comme des matériaux.

\footnotetext{
22 Silbey S.S., 2005, «After legal consciousness », Annual Review of Law and Social Science, vol. 1, p. 323-
} 368. Voir la traduction du texte dans ce numéro. 
Pour mener l'analyse, nous avons identifié :

\section{Grille de lecture}

Le statut des auteurs

La discipline principale (sociologie, science politique, histoire, psychologie sociale)

La problématique de l'article

Les citations LCS et les autres citations en sociologie du droit

Les manières dont les citations LCS sont exploitées - superficiel, discuté, critique, reprise, déférence

La référence à la typologie d'Ewick et Silbey, ou non

L'existence d'une problématisation en termes de pouvoir du droit, voire d'hégémonie juridique

Le « contexte juridique » (espace profane/judiciaire/administratif/etc.)

Le type de données et de méthodes utilisées

Ces questionnements reflètent notre interrogation sur les usages plus ou moins superficiels des travaux LCS, la discussion qu'ils initient (ou non) des résultats et présupposés théoriques et méthodologiques de cet espace de recherche. Nous avons porté attention à la façon dont ces travaux reprennent ou non les problématiques de pouvoir et d'hégémonie du droit, fortement portées par Ewick et Silbey, discutées par exemple dans l'ouvrage New Civil Rights Research de 2006 à partir de plusieurs terrains empiriques mais jamais cités dans l'espace francophone, et au centre du bilan que cette dernière dresse en 2005 dans « After legal consciousness ».

\section{Les LCS : référence plutôt qu'outil de travail}

Cette analyse confirme que les LCS sont rarement mobilisées de façon serrée, mais plutôt comme un ensemble de références légitimant une approche sociologique du droit, à côté d'autres références classiques en sociologie du droit française ou américaine. La référence aux LCS vient appuyer une approche du droit en action, étudiée à travers ses usages ou les rapports que les individus entretiennent avec lui, plutôt que comme un corpus de textes avec lequel on discute. De nombreux usages sont déférents, évoquant la typologie before, with et against the law ou, plus fréquemment, la perspective de considérer le droit au regard des représentations, des catégorisations et des pratiques qu'il suscite, sans recourir à la notion de legality, pourtant centrale pour Silbey (dans ce numéro). Les auteurs qui entament une discussion plus serrée avec les LCS sont souvent liés ou ont été liés au pôle de l'ENS Cachan (Revillard, $2017^{23}$; Touraut, 2014 ; Pillayre, 2014 ; Israël, 2009), même si Durand (2014) ou Lascoumes ou Bezes (2009), par exemple, ont des liens moins directs. Il y a aussi des cas où la jonction avec les LCS n'est pas véritablement faite (ces dernières n'étant pas discutées), malgré les liens évidents qui pouvaient exister (par exemple de Bellaing, 2010, sur le rapport au droit des élèves policiers ou Trémeau, 2017, sur le rapport au droit des jeunes salariés).

\section{L'absence de problématique en termes d'hégémonie}

Un deuxième constat concerne la question du pouvoir et de l'hégémonie. Dans une partie des travaux $\mathrm{LCS}^{24}$, cette question est centrale : non seulement le droit est puissant voire imposant, et offre des cadres d'interprétation, mais ses usages et les manières dont il est construit, vécu et utilisé dans la vie quotidienne dépendent de ressources sociales, de contextes organisationnels, de dispositions et de positions inscrites dans des relations de pouvoir. Plus encore - Silbey insiste fortement sur ce point dans son article

\footnotetext{
${ }^{23}$ Les noms et années mis entre parenthèse renvoient à la bibliographie placée à la fin de l'article, qui constitue ici un matériau, plutôt que des références.

24 Voir Garcia-Villegas M., op.cit., 2003 ; Pélisse J., op.cit., 2005 ou McCann M.W., 2006, « On legal rights consciousness: A challenging analytical tradition », The new civil rights research: A constitutive approach, $\mathrm{p}$. 9-30.
} 
de 2005 - la légalité telle qu'elle est construite dans la vie de tous les jours à travers les trois formes de rapports au droit (before, with et against) est fondamentalement structurante et hégémonique. Nos relations sociales et l'ensemble des sphères dans lesquelles nous évoluons seraient marquées par une idéologie juridique en partie contradictoire mais dont les contradictions en soutiennent l'hégémonie. Cette thèse d'une légalité hégémonique n'apparaît pas dans notre corpus. La question du pouvoir du droit y est présente, mais pas celle de son hégémonie dans la vie quotidienne, comme le montre la rareté de la notion de légalité (legality) dans les vingt articles étudiés. Comme aux Etats-Unis, une lecture individualisante, centrée soit sur la typologie de Ewick et Silbey, soit sur la perspective bottom-up propre aux LCS, a nettement dominé cette phase d'appropriation en France.

\section{Une approche du droit peu "décentrée »}

Ce constat fait écho à la forte représentation d'articles à la frontière de la sociologie et de la science politique, dans le sillage des appels à une sociologie politique du droit émanant de J. Commaille. Beaucoup de travaux qui font référence aux LCS empruntent ainsi à la sociologie des mobilisations, faisant écho aux travaux de McCann: ils s'intéressent de fait moins au droit dans la vie ordinaire qu'à son usage spécifique dans le cadre d'actions collectives. De même, plusieurs articles portent sur un ensemble de règles juridiques ou un processus de régulation qui part de l'Etat dont on analyse la mise en œuvre ou les effets - une problématique classique de science politique (Vidal, 2009 ; N’Diaye, 2016 ; Commaille, 2016).

La plupart des articles de notre corpus s'inscrivent même dans une perspective de claiming, où l'horizon judiciaire est une perspective plus ou moins proche: de Galembert (2015) et la cause du voile, Fillion et Torny (2015) sur la réparation judiciaire des victimes du distilbène, Jouzel et Prete (2014) sur les victimes des pesticides, Lejeune et Oriane (2014) concernant la strategic litigation en matière de discrimination, Guillaume (2015) sur les syndicats anglais, etc. Le droit est manié dans un horizon stratégique, comme une ressource manipulable plus qu'un cadre de pensée hégémonique, même si cela n'empêche pas de prendre en compte ses effets cognitifs sur la définition de la situation, des responsabilités et des torts.

La perspective d'étudier le droit hors des institutions ou dans la vie quotidienne apparaît ainsi relativement éloignée, la plupart des références de notre corpus se centrant sur les interactions entre des profanes, intermédiaires et/ou professionnels du droit, à l'intérieur d'espaces pré-judiciaires, judiciaires ou administratifs. Certains articles adoptent néanmoins un point de vue plus fortement décentré, s'intéressant au droit hors des lieux institutionnels, que ce soit Revillard (2017) sur l'appropriation des politiques du handicap (sur le modèle d'Engel et Munger), Schijman (2013) sur les usages du logement social en Argentine, Denis et Pontille (2013) sur les internautes participant à la constitution des cartes géographiques ou Lascoumes et Bezès (2009) concernant les représentations ordinaires de la corruption en France. Aucun n'entre toutefois directement en discussion avec la typologie d'Ewick et Silbey, à l'exception du dossier dirigé par Barrault-Stella et Spire (2017) qui revient à partir de travaux empiriques originaux, après les critiques énoncées plus de dix ans avant, sur l'absence d'ancrage social du modèle before/with/against the law chez Ewick et Silbey.

\subsection{Une effervescence récente autour des LCS et de la question du pouvoir}


De la soutenance de plusieurs thèses les utilisant plus ou moins centralement (Kubiak ${ }^{25}$, Vincent $^{26}$ et Juston ${ }^{27}$ en 2016 ; Pillayre ${ }^{28}$ et Trémeau $^{29}$ en 2017 par exemple), à la journée des doctorants du RT13 de Sociologie du droit et de la Justice de l'Association française de sociologie en mars 2018, qui reprend l'image de couverture de The Common Place of Law ; de la traduction de l'ouvrage d'Engel et Munger (déjà cité) à la publication de numéros spéciaux comme ceux de Sociétés contemporaines en 2017 ou ce même dossier de Droit et Société, une certaine effervescence existe néanmoins récemment autour des LCS en France. Si notre analyse bibliométrique montrait une baisse du nombre de références après 2014, plusieurs indices montrent que la diffusion des LCS se poursuit et s'intensifie sans doute récemment. Certes, les références sont parfois cosmétiques, et c'est souvent via la question de la socialisation au droit et au détour d'un chapitre que les LCS sont mentionnées et parfois discutées.

Mais cette diffusion traduit selon nous la reconnaissance institutionnelle d'une perspective qui a profondément renouvelé la sociologie du droit américaine, et française depuis une quinzaine d'années. Leur place dans des manuels récents ${ }^{30}$, un texte de synthèse sur les évolutions de la sociologie du droit française ${ }^{31}$, ou dans le dernier ouvrage de $\mathrm{J}$. Commaille ${ }^{32}$ en témoignent. Cette institutionnalisation conduit aussi à discuter cette perspective, à l'utiliser non pas seulement avec déférence ou pour signaler une approche du droit en actes, mais aussi avec l'ambition d'en analyser les limites, les présupposés ou la nécessité d'aller plus loin. Certains travaux proposent par exemple de l'utiliser en spécifiant les rapports et usages du droit des dominants, comme on l'a déjà signalé. D'autres proposent de compléter la typologie de Ewick et Silbey à partir d'une étude des représentations ordinaires du droit via l'usage de focus group ${ }^{33}$, de critiquer ses aspects trop mentalistes en invitant à une jonction avec une approche pragmatiste ${ }^{34}$ ou de les employer pour étudier l'action publique ${ }^{35}$. En somme, les usages des LCS sont variés, pluriels, rarement frontaux et critiques vis-à-vis de cette perspective de recherche, même si, en 2017 et 2018, une certaine effervescence traduit une mise en discussion plus directe qui se manifeste fortement dans ce dossier de Droit et société.

\section{Conclusion}

\footnotetext{
${ }_{25}$ Kubiak J., 2016, La" managérialisation" de la prévention des risques professionnels en entreprise. Enquête parmi les préventeurs de la SNCF, Thèse de sociologie, Univ. Versailles Saint Quentin en Yvelines.

26 Vincent F., 2016, Un temps qui compte. Une sociologie ethnographique du travail « en 12 heures » à l'hôpital public, Thèse de sociologie, Univ. Paris Dauphine.

27 Juston R., 2016, Le corps médico-légal. Les médecins légistes et leurs expertises, Thèse de sociologie, Univ. Versailles Saint Quentin en Yvelines.

28 Pillayre H., 2017, Justice et justesse de l'indemnisation. Acteurs et dispositifs de l'État providence à l'épreuve du scandale de l'amiante, Thèse de sociologie, EHESS.

29 Tremeau C., 2017, S'informer, s'indigner, réclamer, revendiquer ou non en entreprise: les jeunes salariés à l'épreuve de leurs droits, Thèse de sociologie, Univ.de Nantes.

30 Voir Delpeuch T., Dumoulin L., Galembert Claire de, 2014, Sociologie du droit et de la justice, Paris, Armand Colin ; Christin A. et Ollion E., 2012, La sociologie aux États-Unis aujourd'hui, Paris, La Découverte.

${ }^{31}$ Israël L., 2013, « Legalize it! The rising place of law in French sociology », International Journal of Law in Context, 9 (2), p. 262-278.

32 Commaille J., 2015, A quoi nous sert le droit?, Paris, Gallimard.

33 Dumoulin L, Vigour C., 2017, "A focus group approach to analyzing experiences of law and justice », communication au colloque Law and Society, Mexico.

${ }^{34}$ Chappe V.-A., 2013, L'égalité en procès. Sociologie politique du recours au droit contre les discriminations au travail, Thèse de doctorat en sciences sociales, ENS Cachan.

35 Voir par exemple Baudot P.-Y., Revillard A., 2014, « Entre mobilisations et institutions. Les politiques des droits dans l'action publique », Gouvernement et action publique, vol. 4 (4), p. 9-33.
} 
Laissant de côté les questions de traduction de concepts culturellement ancrés dans une société singulière ${ }^{36}$, ainsi que la réception des LCS aux Etats-Unis ou dans d'autres espaces académiques ${ }^{37}$, cet article a tenté de montrer comment avait été importée et s'était diffusée cette perspective de recherche en France. En conclusion, quelques pistes de recherche peuvent alors être rapidement esquissées. Une première piste part de ce constat selon lequel les LCS sont souvent réduites à l'utilisation de la typologie fondatrice d'Ewick et Silbey sur les rapports au droit, notamment dans une lecture stratégique de l'action individuelle ou collective. Une prise en compte plus fidèle nécessiterait, selon nous, de partir "à l'envers », c'est-à-dire de la place du droit dans la vie quotidienne. Il reste en effet beaucoup à faire pour documenter l'emprise du droit dans les différents sous-champs de la vie sociale. A quel point est-il omniprésent, hégémonique? Sous quelle forme s'exprime-t-il et est-il construit, représenté, vécu ? Les LCS peuvent orienter vers un questionnement en termes de prégnance de la légalité, faisant même de celle-ci une hypothèse où nous vivrions dans des sociétés hyper juridicisées et dans l'obligation de composer avec les normes juridiques.

Ce postulat n'a rien d'évident, en réalité, et les travaux des Engel sur la Thaïlande l'ont montré38. Il n'est pas forcément pertinent de parler d'hégémonie du droit et il faut sans doute tenir ensemble des perspectives horizontales autant que verticales pour étudier les consciences du droit ${ }^{39}$. Une question importante est ainsi de se demander comment le droit s'articule avec la légalité ordinaire, qui incorpore bien d'autres logiques et normativités. Ne faut-il pas distinguer les différentes sphères de la vie sociale, où les catégories juridiques s'imposent de façon plus ou moins régulière, plus ou moins totale, aux pratiques et interactions sociales ? Il s'agit de ne pas se faire piéger par la dimension totalisante de la notion d'hégémonie, pour repenser dans une perspective plus pragmatiste les degrés, les variations, les contrastes ou les tensions au sein du processus de juridicisation. Il s'agit également de penser des phases silencieuses du droit, où celuici est en arrière-plan sous la forme d'un "inconscient grammatical » 40 qui structure ce que Silbey nomme legality. Dans un certain nombre de situations, le droit ne participe pas explicitement à la régulation des situations. Mais il est toujours un potentiel de l'interaction, un horizon possible vers lequel celle-ci pourrait être amenée à se déployer. Il faut alors explorer les conditions de juridicisation, non d'une société ou d'un système social, mais d'une situation ${ }^{41}$ : comment le droit est-il défini et pourquoi est-il invoqué ? Quelles sont les conditions de son «passage $»^{42}$ ? Cela engage à un travail microsociologique sur les dynamiques interactionnelles, notamment en situation de dispute.

\footnotetext{
36 Voir des éléments dans Israël et Pélisse, op.cit. et Pélisse, op.cit., 2005. Les LCS sont fortement internationalisées aujourd'hui, comme le montre la diversité des terrains géographiques qui mobilisent cette perspective, que ce soit dans notre corpus ou hors de l'espace francophone. Dans les deux panels dédiés aux LCS lors du dernier congrès de la Law and Society en 2017, la moitié des intervenants n'étaient pas américains.

37 Cela n'est pas sans poser problème tant cette réception a aussi une histoire aux Etats-Unis et ailleurs. Voir Pélisse, op. cit., 2005, qui discute par exemple les travaux de M. Hertogh, chercheur néerlandais qui revendique une approche européenne des LCS distincte de celle théorisée par Ewick et Silbey.

38 Voir D.M. Engel et J. S. Engel, Tort, Custom and Karma. Globalization and Legal Consciousness in Thailand, Stanford University Press, 2010.

${ }^{39}$ Voir D.M. Engel, « Vertical and Horizontal Perspectives on Rights Consciousness », Indiania Journal of

Global Legal Studies, vol. 19, issue 2, p. 423-455.

${ }^{40}$ Lemieux C., 2009, Le devoir et la grâce, Paris, Economica.

41 Comme le propose par exemple Dulong R., 1991, « «On n’a pas le droit...». Sur les formes d'appropriation du droit dans les interactions ordinaires», in F. Chazel et J. Commaille (éd.), Normes juridiques et régulation sociale, Paris, LGDJ, p. 257-264.

42 Pour reprendre un terme employé par B. Latour ou J.-M. Weller.
} 
Une deuxième voie d'exploration porte sur les acteurs, les formes et la matérialité de la légalité. L'affirmation de l'hégémonie du droit laisse dans l'ombre la question des dispositifs par lesquels celui-ci est capable de s'exprimer. Nous ne désignons pas ici uniquement les appareils formels de la police et de la justice, mais plus largement toutes les formes de médiation par lesquelles le droit se rappelle comme acteur potentiel de notre existence. Ces médiations peuvent être incarnées par des «intermédiaires du droit» qui n'en sont pas uniquement les professionnels ${ }^{43}$. Mais ces médiations sont également disséminées dans notre environnement quotidien, à travers des produits du droit (affiches, signalements, règlements, etc.) qui rappellent indirectement la légalisation de nos quotidiens, à l'instar des « outil de gestion ${ }^{44}$ et de "négociation ${ }^{45}$ qui habitent le monde du travail. La légalité émerge également aux points de rencontre entre humains et objets qui habitent les espaces sociaux. Ces quelques pistes appellent à saisir les LCS non pas comme un paradigme par rapport auquel il faudrait se positionner, mais comme un ensemble de ressources théoriques, critiquables et ouvertes aux hybridations, afin de penser la présence de la légalité et l'action $d u$ droit et par le droit dans nos sociétés.

\section{Annexe : les vingt articles étudiés}

Barrault-Stella Lorenzo et Spire Alexis, 2017, «Introduction », Sociétés contemporaines, $\mathrm{n}^{\circ} 108$, p. 5-14.

Beal Arnaud, Kalampalikis Nikos, Fieulaine Nicolas et Haas Valérie, 2014, "Expériences de justice et représentations sociales: l'exemple du non-recours aux droits», Les Cahiers Internationaux de Psychologie Sociale, $\mathrm{n}^{\circ} 103, \mathrm{n}^{\circ} 3$, p. 549-573.

Bellaing Cédric Moreau de, 2010, « De l'obligation à la ressource », Déviance et Société, 34 (3), p. 325-346.

Commaille Jacques, 2016, «Les enjeux politiques d'un régime de connaissance sur le droit. La sociologie du droit de Georges Gurvitch », Droit et société, n 94, p. 547-564.

Denis Jérôme et Pontille David, 2013, "Une infrastructure élusive », Réseaux, n 178-179, p. 91-125.

Durand Corentin, 2014, «Construire sa légitimité à énoncer le droit. Étude de doléances de prisonniers », Droit et société, n 87, p. 329-348.

Fillion Emmanuelle et Torny Didier, 2015, « De la réparation individuelle à l'élaboration d'une cause collective », Revue française de science politique, 65 (4), p. 583-607.

Galembert Claire de, 2016, « Le droit à porter le voile: cause perdue ou naissance d'une politics of rights? », Revue interdisciplinaire d'études juridiques, 75 (2), p. 91-114.

Guillaume Cécile, 2015, « Les syndicats britanniques et le recours au contentieux juridique », $L a$ nouvelle revue du travail, $\mathrm{n}^{\circ} 7$ (en ligne http://nrt.revues.org/2354).

Israël Liora, 2009, « Résister par le droit ? », L’Année sociologique, 59 (1), p. 149-175.

\footnotetext{
43 Voir Talesh S., Pélisse J., 2018, « How Legal Intermediaries Facilitate or Inhibit Social Change », LIEPP Working Paper, ${ }^{\circ} 73$, et Pélisse J., "Travailler le droit: lectures et perspectives sociologiques », Revue française de sociologie,2018/1 (vol. 59), p. 99-125.

${ }^{44}$ Voir Chiapello E. et Gilbert P., 2013, Sociologie des outils de gestion : introduction à l'analyse sociale de l'instrumentation de gestion, Paris, La Découverte.

45 Chappe V.-A., 2017, «L'agentivité d'un outil de quantification des inégalités sexuées en entreprise », Working Paper i3. https://hal-mines-paristech.archives-ouvertes.fr/hal-01667367/document [Consulté le 29 janvier 2018].
} 
Jouzel Jean-Noël et Prete Giovanni, 2014, « Devenir victime des pesticides. Le recours au droit et ses effets sur la mobilisation des agriculteurs Phyto-victimes », Sociologie du travail, 56 (4), p. 435-453.

Lascoumes Pierre et Bezes Philippe, 2009, «Les formes de jugement du politique ", L'Année sociologique, 59 (1), p. 109-147.

Lejeune Aude et Orianne Jean-François, 2014, "Choisir des cas exemplaires: la Strategic litigation face aux discriminations », Déviance et Société, 38 (1), p. 55-76.

N’Diaye Marième, 2016, "La réforme de la Moudawana: Une révolution? Lire la norme islamique à l'aune de sa redéfinition par l'État », Studies in Religion/Sciences Religieuses, 45 (2), p. 146-165.

Pillayre Héloïse, 2014, «Les victimes confrontées à l'incertitude scientifique et à sa traduction juridique : le cas du vaccin contre l’hépatite B », Droit et société, n ${ }^{\circ} 86$, p. 33-53.

Revillard Anne, 2017, "La réception des politiques du handicap: une approche par entretiens biographiques », Revue française de sociologie, 58 (1), p. 71-95.

Schijman Emilia, 2013b, «Usages, pactes et "passes du droit» », Déviance et Société, 37 (1), p. 51-65.

Touraut Caroline, 2014, «Les proches de détenus et leurs rapports ordinaires au droit pénitentiaire », Droit et société, n ${ }^{\circ}$ 7, p. 375-392.

Trémeau Camille, 2017, «De jeunes salariés confrontés à l'(in)justice du travail : recours aux prud'hommes et effets socialisateurs de l'épreuve judiciaire », Politix, n 118, p. 157-181.

Vidal Dominique, 2009, «Une relation ancillaire à l'épreuve du droit », Travail, genre et sociétés, $\mathrm{n}^{\circ} 22$, p. 97-113. 